Supporting Information For

\title{
Phase Engineering of Epitaxial Stanene on Surface Alloy
}

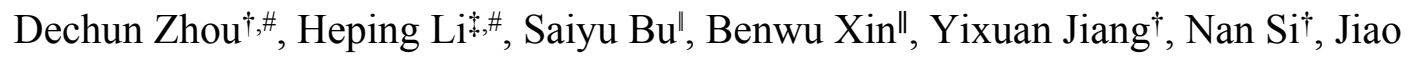

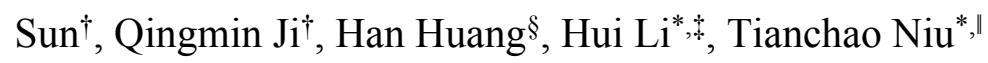

$\dagger$ Herbert Gleiter Institute of Nanoscience, School of Material Science and Engineering, Nanjing University of Science \& Technology, Nanjing 210094, China \$ Beijing Advanced Innovation Center for Soft Matter Science and Engineering, Beijing University of Chemical Technology, Beijing 100029, China $\S$ Hunan Key Laboratory of Super-Microstructure and Ultrafast Process, College of Physics and Electronics, Central South University, Changsha 410083, China $\|$ School of Mechanical Engineering, Shanghai Jiao Tong University, Shanghai 200240, China

* Corresponding Authors:

hli@buct.edu.cn (H. L.); niutianchao1415@sjtu.edu.cn (T. N.)

\# D. Z. and H. L. contributed equally to this work. 


\section{Experimental and Computational Methods}

Deposition of Sn. Au(111) single crystal was cleaned by cycles of Ar ion sputtering $\left(1.1 \mathrm{keV}, 1.5 \times 10^{-5} \mathrm{mbar}\right)$ and e-beam heating in ultrahigh vacuum at $660 \mathrm{~K}$. The surface was characterized by STM to show the typical herringbone reconstruction. ${ }^{1}$ The $\mathrm{Au}_{2} \mathrm{Sn}$ alloy surface was prepared by deposition of $\mathrm{Sn}$ onto the clean $\mathrm{Au}(111)$ surface followed by annealing at $600 \mathrm{~K}$ for $20 \mathrm{~min}$. The surface structure and uniformity was measured by STM before the further deposition of Sn. Then, Sn was deposited onto $\mathrm{Au}_{2} \mathrm{Sn}(111)$ surface at RT from a well-degassed boron nitride crucible (1073 K). To reveal the growth mechanism and explore the intermediates, the substrate temperature during deposition and annealing temperature after deposition were delicately controlled.

Scanning Tunneling Microscopy. The STM experiments were performed using an SPECS JT-SPM equipped with a Nanonis controller in an operating pressure of $2 \times$ $10^{-10}$ Torr. $^{1}$ The STM images were obtained at liquid nitrogen temperature $(77 \mathrm{~K})$ with electrochemically etched W tips in a constant current mode. STS measurements were collected using a lock-in amplifier by applying a small periodic modulation to the applied voltage $(15 \mathrm{mV}, 833 \mathrm{~Hz})$.

Computational Details. DFT calculations were performed with the Vienna Ab initio Simulation Package (VASP) ${ }^{2}$ using the Perdew-Burke-Ernzerh (PBE) functional ${ }^{3}$ in conjunction with the projector-augmented-wave (PAW) pseudopotential and a plane wave basis set $\left(\mathrm{E}_{\text {cutoff }}=300 \mathrm{eV}\right)$. The van der Waals correction was also included in the DFT calculations. In order to obtain accurate atomic structure, four layers of $\mathrm{Au}$ substrates were used, where the bottom two layers of gold are fixed, and the atoms of the stanene layer and the topmost two layers of $\mathrm{Au}_{2} \mathrm{Sn}$ substrate are fully relaxed. $\mathrm{A}$ vacuum space of $15 \AA$ along the $z$ direction was applied to eliminate the image interaction. The structures were relaxed until the maximum atomic force is $<0.01 \mathrm{eV} / \AA$. The $3 \times 3 \times 1 \mathrm{k}$-point grids were used in the optimization of the stanene on substrate. The band structure of epitaxial stanene was derived at the PBE+SOC level with the $20 \times 20 \times 1$ k-point grids. 

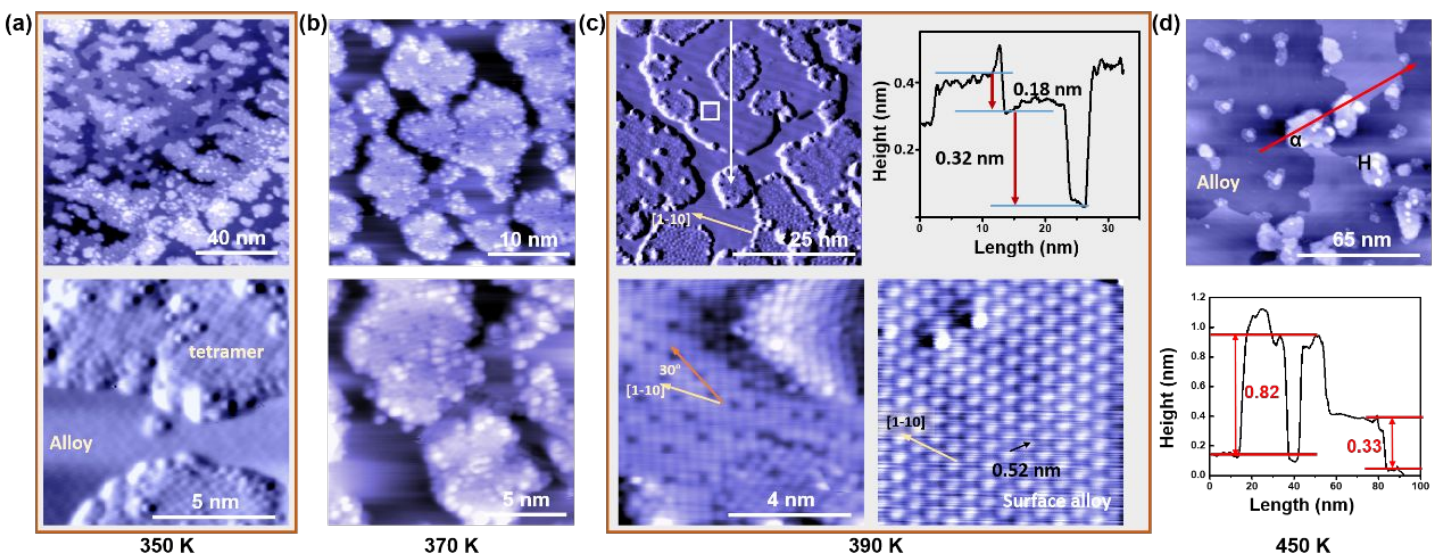

Fig. S1. Substrate-temperature-dependent surface morphology of direct deposition onto $\mathrm{Au}_{2} \mathrm{Sn}(111)$ alloy. The temperature is indicated under each panel, respectively. Scanning parameters: (a) $1.4 \mathrm{~V}, 100 \mathrm{pA}$ (top); $400 \mathrm{mV}, 400 \mathrm{pA}$ (bottom); (b) $1.2 \mathrm{~V}$, 100 pA (both panels); (c) $500 \mathrm{mV}, 300$ pA (top left); $10 \mathrm{mV}, 770$ pA (bottom left); 10 $\mathrm{mV}, 2.2 \mathrm{nA}$ (bottom right); (d) $1.8 \mathrm{~V}, 80 \mathrm{pA}$

At relatively low substrate temperature, direct deposition leads to larger Sn island than those obtained under high temperature (Figure S1a and 1b). Meanwhile, the Sn tetramer and vacancy dominate the whole surface species (Figure S1a and 1b). It is noted that high temperature induces the apparent generation of $\mathrm{Au}_{2} \mathrm{Sn}$ alloy islands adjacent to the Sn aggregates (Figure S1c). The $\alpha$-stanene starts to evolve above $450 \mathrm{~K}$. It has a height of $\sim 0.82 \mathrm{~nm}$, and prefers to be generated at the edges (Figure S1d). 

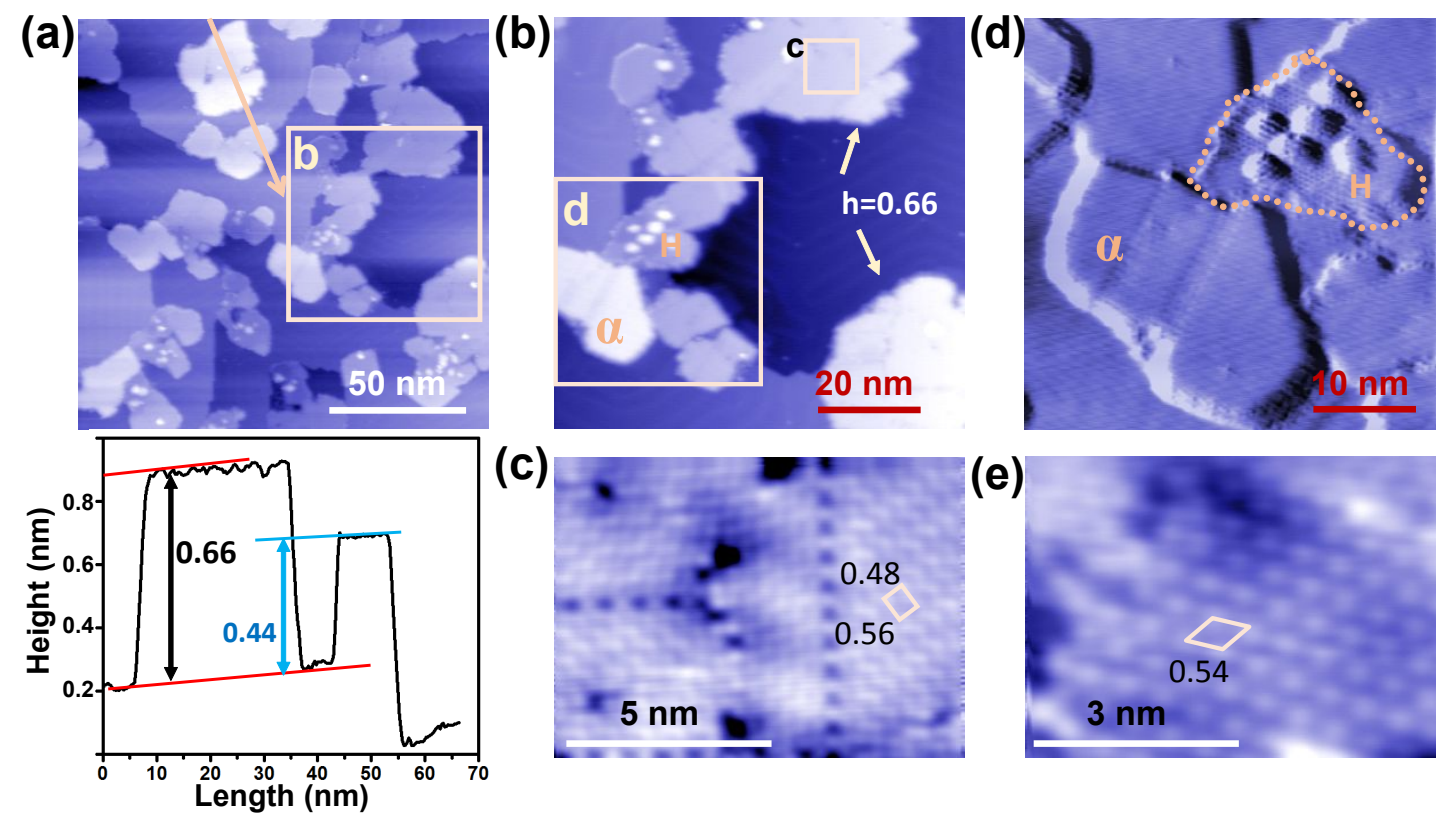

Fig. S2. Annealing at $450 \mathrm{~K}$ for $20 \mathrm{~min}$. The $\mathrm{Sn}$ thin films were firstly prepared by direct deposition of $\mathrm{Sn}$ onto the $\mathrm{Au}_{2} \mathrm{Sn}(111)$ held at $390 \mathrm{~K}$. Scanning parameters: (a) $1.5 \mathrm{~V}, 120 \mathrm{pA}$; (b) -1 V, 100 pA; (c) $-10 \mathrm{mV}, 2.2 \mathrm{nA}$; (d) $-100 \mathrm{mV}, 400$ pA; (e) -20 $\mathrm{mV}, 800 \mathrm{pA}$

Nearly $90 \%$ of the Sn domains transits to $\alpha$-phase after annealing at $450 \mathrm{~K}$ for 20 min. Figure S2a shows a large-scale STM image of the distribution of Sn islands. The island located inside of the terrace is $0.22 \mathrm{~nm}$ higher than those at the step edges, but features the identical $\alpha$-phase. The height difference corresponds to an atomic layer underneath. There is abundance of linear boundaries within each $\alpha$-stanene island. The zoom-in scans reveal the presence of hexagonal dot arrays near the step edges. 

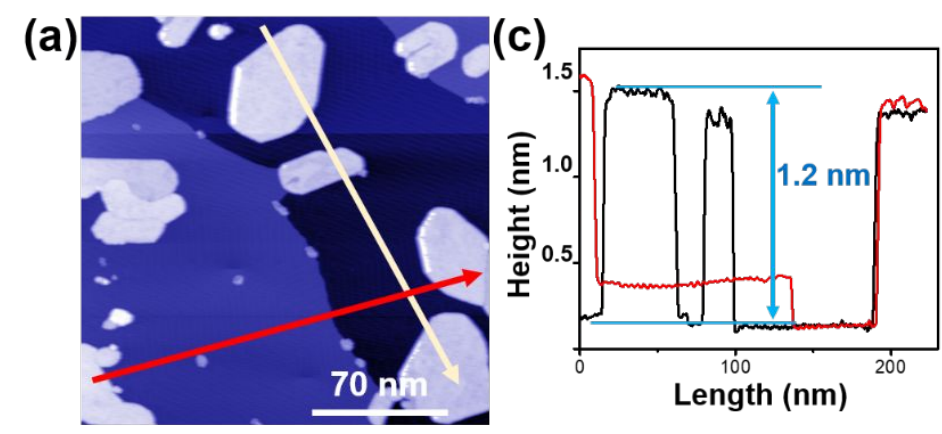

(e)
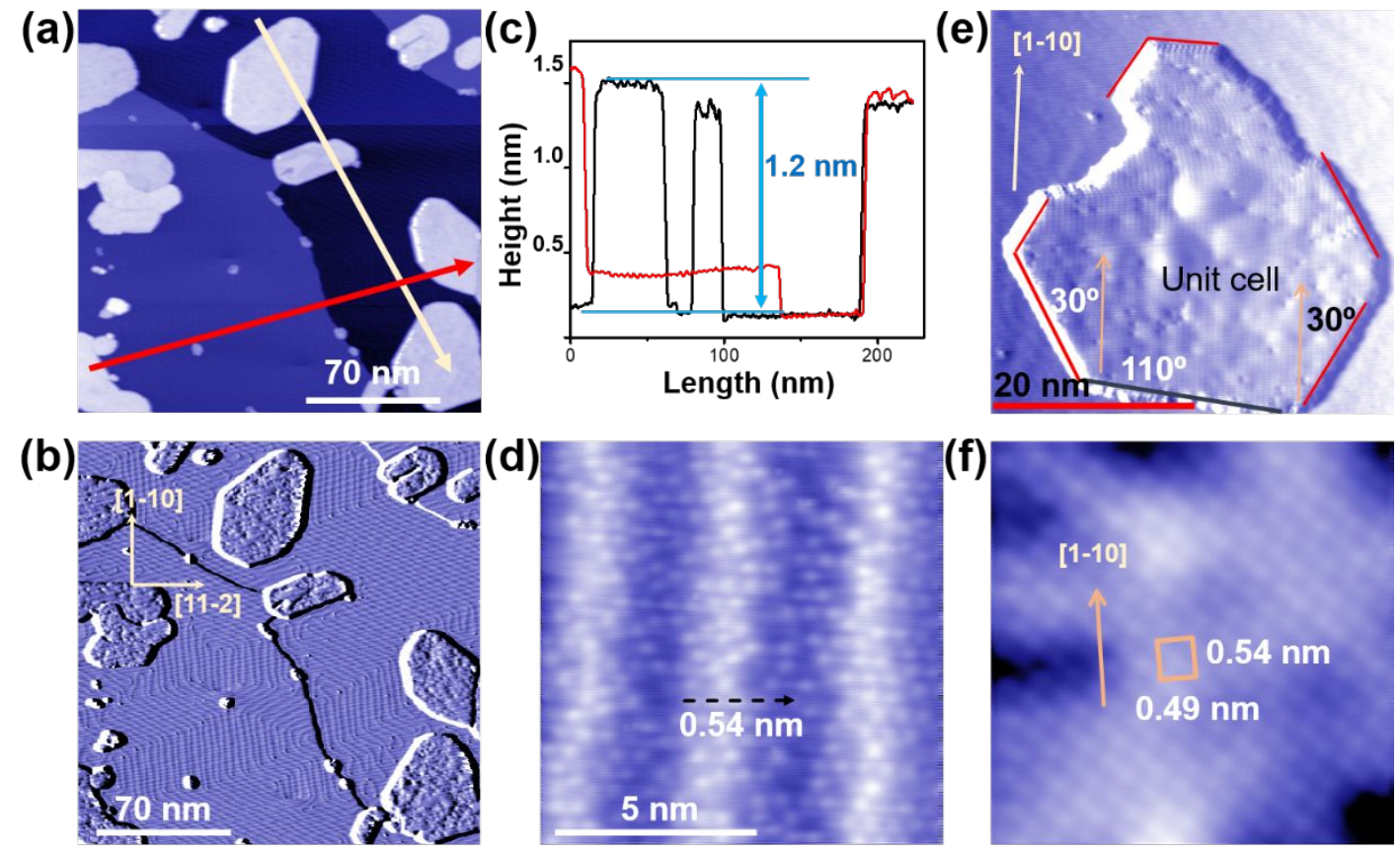

Fig. S3. Further annealing the sample shown in Figure S2 to $530 \mathrm{~K}$. All the Sn islands transform to the $\alpha$-phase, leaving the bare area with $\mathrm{Au}_{2} \mathrm{Sn}$ (111) surface alloy. Scanning parameters: (a) and (b) $1.6 \mathrm{~V}, 60$ pA; (d) $20 \mathrm{mV}, 2 \mathrm{nA}$; (e) $300 \mathrm{mV}, 500$ pA; (f) $20 \mathrm{mV}, 500 \mathrm{pA}$

It is quite interesting to note that annealing at $530 \mathrm{~K}$ leads to the complete transformation to the $\alpha$-phase. Measured line-profiles present an average height of 1.2 $\mathrm{nm}$ for the $\alpha$-stanene. This value is much larger than the $\alpha$-stanene prepared at low temperature $(0.66 \sim 0.8 \mathrm{~nm})$. Furthermore, in contrast to other conditions that $530 \mathrm{~K}$ annealing generates wholly the $\beta$-stanene, the ultrastable $\alpha$-phase should be ascribed to the presence of a buffer layer between the alloy surface and stanene. The herringbone reconstruction of $\mathrm{Au}(111)$ in bare area is still visible, while the short-range ordered bright atoms exhibit an interatomic distance of $0.54 \mathrm{~nm}$. The edges of $\alpha$-stanene island are either sharp or rough that depend on the rotation angle with respect to the lattice of stanene. Atomically resolved STM image in Figure S3f shows a rectangle unit cell of $0.49 \mathrm{~nm}$ by $0.54 \mathrm{~nm}$, whereas the dark and bright areas are due to the modulation by underlying buffer layer. 
(a)

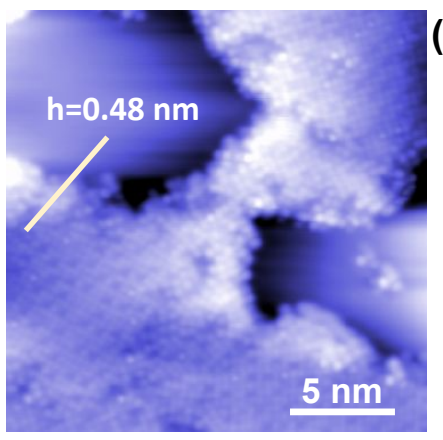

(b)

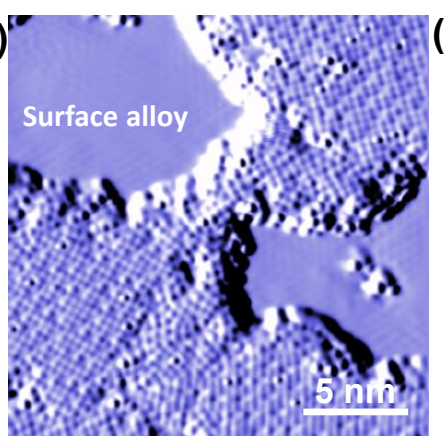

(c)

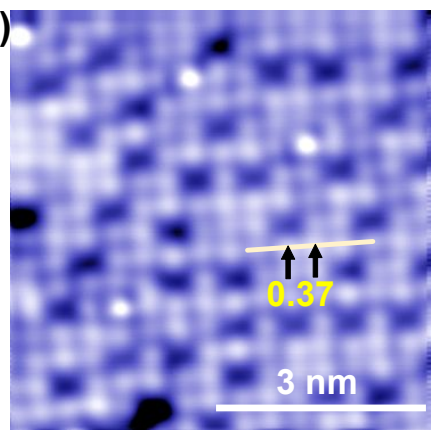

Fig. S4. Direct deposition of Sn onto $\mathrm{Au}_{2} \mathrm{Sn}(111)$ held at $330 \mathrm{~K}$, followed by annealing at $370 \mathrm{~K}$ for $20 \mathrm{~min}$. Scanning parameters: (a) and (b) $150 \mathrm{mV}, 1 \mathrm{nA}$; (c) $-120 \mathrm{mV}, 1$ $\mathrm{nA}$

(a)

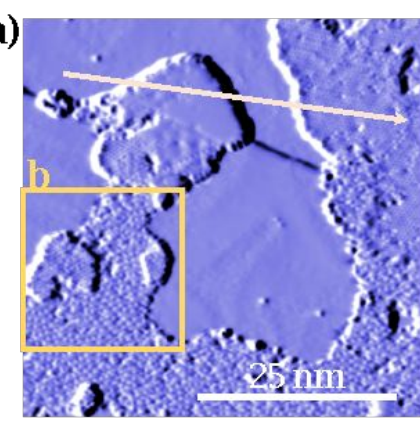

(b)
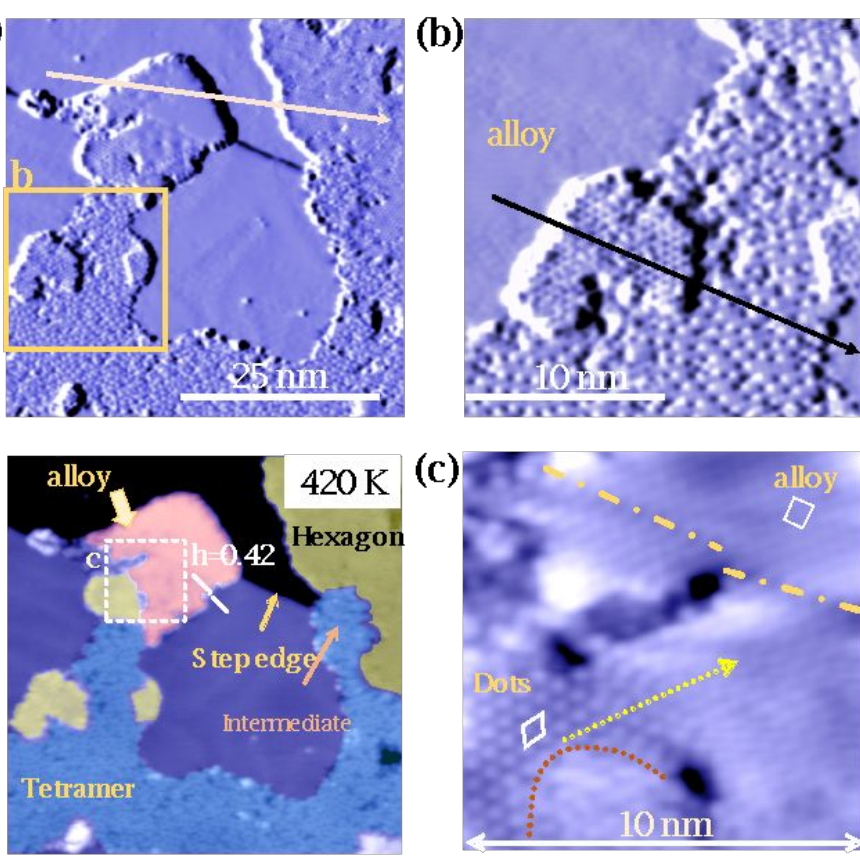

(c)

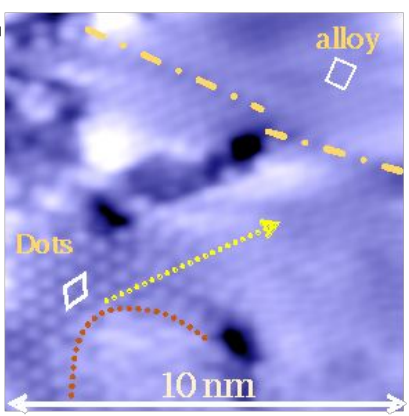

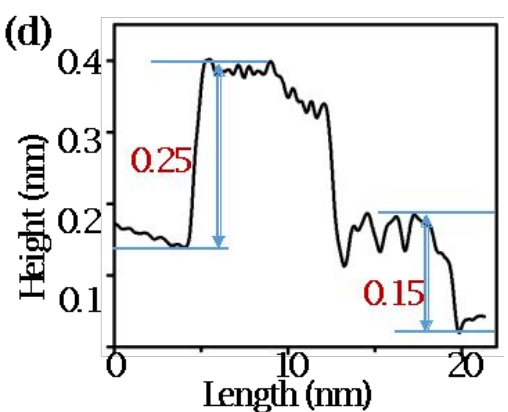

(e)

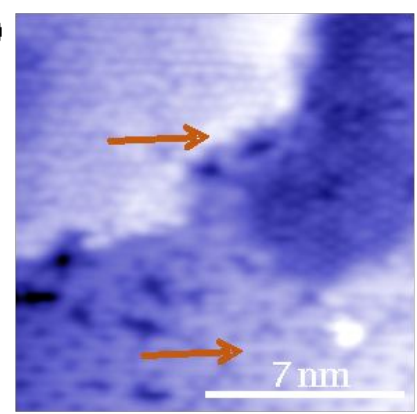

Fig. S5. Further annealing the sample shown in Figure S4 at $420 \mathrm{~K}$ for $20 \mathrm{~min}$. Scanning parameters: (a) $600 \mathrm{mV}, 200 \mathrm{pA}$; (b) $600 \mathrm{mV}, 800 \mathrm{pA}$; (c) $100 \mathrm{mV}, 1 \mathrm{nA}$; (e) $200 \mathrm{mV}$, $800 \mathrm{pA}$

Phase transition occurs at $420 \mathrm{~K}$ including the generation of hexagonal dot arrays and $2 \times \sqrt{3}$ surface alloy. It is noted that the $\alpha$-phase emanates from the substrate steps showing a rectangular shape. The height of alloy is $0.74 \mathrm{~nm}, 0.3 \mathrm{~nm}$ higher than the hexagonal dot arrays, signaling the presence of a buffer layer underneath. Due to the 
low substrate temperature, there are randomly distributed vacancies within the alloy domain. The vacancy is one missing atom with a size comparable to the Sn tetramer vacancy that can be considered as the precursor. Meanwhile, annealing also generates hexagonal dot arrays at the edges of $\mathrm{Sn}$ islands which are unidirectionally aligned with the densely packed direction of Sn tetramers.

(a)

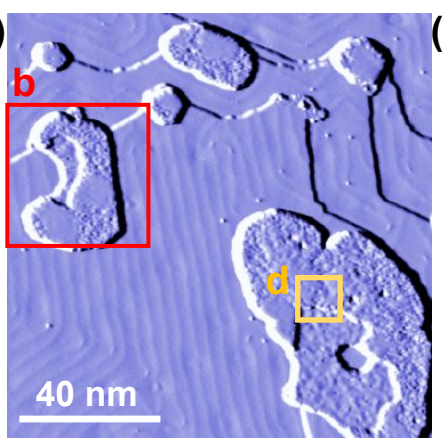

(d)

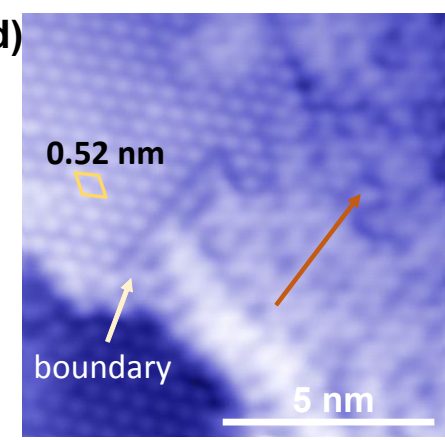

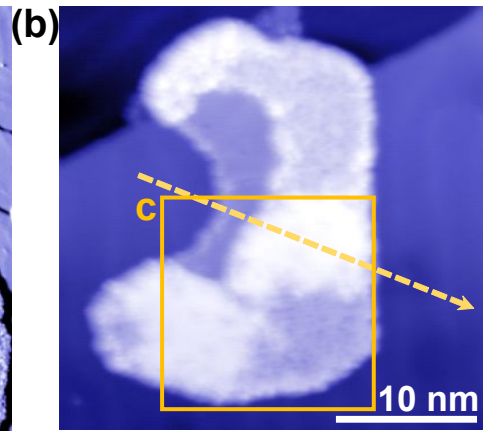

(c)

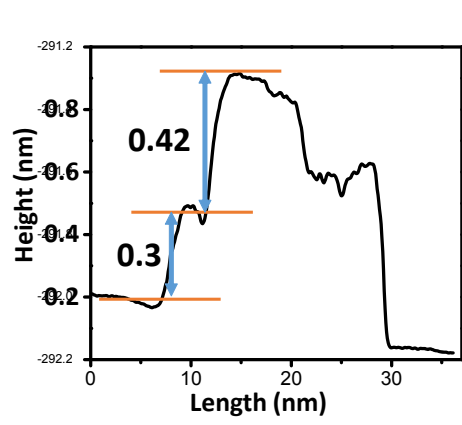

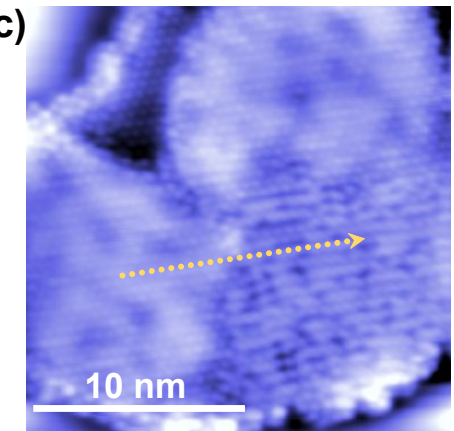

$=\mathrm{C1}$

Fig. S6. Increasing the annealing temperature to $470 \mathrm{~K}$ and maintaining for $30 \mathrm{~min}$. Tetramer and vacancies wholly disappeared, which transit to hexagonal dot arrays and $\beta$-stanene. ${ }^{4}$ Scanning parameters: (a) $1.8 \mathrm{~V}, 80$ pA; (b) 1 V, 200 pA; (c) 400 mV, 600 $\mathrm{pA}$; (d) $100 \mathrm{mV}, 1 \mathrm{nA}$
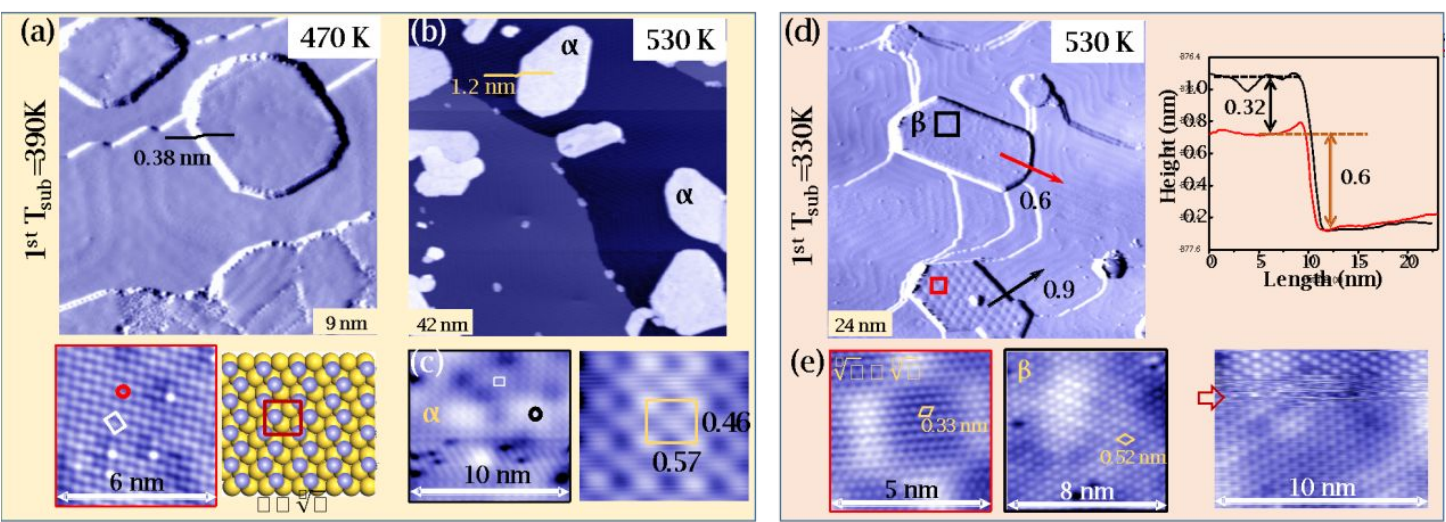

Fig. S7. (a) Keep the substrate at $390 \mathrm{~K}$ at the first deposition of Sn, followed by 
annealing at $470 \mathrm{~K}$, leading to the $2 \times \sqrt{3}$ surface alloy; and (b) $530 \mathrm{~K}$, generating the $\alpha$-stanene; (c) Zoom-in scan of the bright island in (b), showing a two-fold symmetry and a rectangle unit cell; (d) Substrate temperature is $330 \mathrm{~K}$ during the initial deposition. Further annealing at $530 \mathrm{~K}$ leads to a hexagonal phase and a Moiré pattern. The line profiles are taken across the hexagonal (black) and Moiré islands (red). (e) Atomically resolved STM images of (from left to right) hexagonal phase, Moiré pattern and tip-change on the hexagonal phase. Scanning parameters: (a) $500 \mathrm{mV}, 100 \mathrm{pA}$ (top); $100 \mathrm{mV}, 800$ pA (bottom); (b) $1.6 \mathrm{~V}, 60$ pA (c) $-50 \mathrm{mV}, 600$ pA; (left: large-area STM image; right: atomically resolved STM image) (d) $2.2 \mathrm{~V}, 80 \mathrm{pA}$; (e) $-300 \mathrm{mV}, 600 \mathrm{pA}$ (left); $200 \mathrm{mV}, 600 \mathrm{pA}$ (middle) and $100 \mathrm{mV}, 200$ pA (right).

Figure S7a shows the $\mathrm{Sn}$ islands after annealing at $470 \mathrm{~K}$. Atomically resolved STM image reveals a zigzag pattern with a rectangle unit cell of $0.58 \times 0.5 \mathrm{~nm}^{2}$. Although the lattice size is quite similar with the $\alpha$-stanene (4.73 $\AA, 5.85 \AA$ ), the layer thickness is only $0.38 \mathrm{~nm}$ that is much lower than the puckering height of $\alpha$-stanene. The packing structure of one-atomic layer $\mathrm{Sn}$ atoms is depicted to be a $2 \times \sqrt{3}$ superstructure $^{5}$ (atomic model in low panel, Figure S7a). Further annealing the sample to $530 \mathrm{~K}$ results in as well a two-fold symmetric lattice but with a much larger layer thickness $(1.2 \mathrm{~nm})$ than the $2 \times \sqrt{3}$ superstructure, which can be assigned to the $\alpha$-stanene (Figure S7b). Panels in Figure S7c are high-resolution STM images showing a rectangle unit cell of $0.46 \times 0.57 \mathrm{~nm}^{2}$ that is close to $\alpha$-stanene $(4.73 \AA, 5.85 \AA)$. The much larger layer thickness suggests the presence of a wetting layer between stanene and substrate.

Figure S7d demonstrates two different phases exhibiting layer thickness of $0.6 \mathrm{~nm}$ and $0.92 \mathrm{~nm}$, respectively (line profiles). Zoom-in scans (Figure S7e) are taken in different phases as marked in Figure S7d. 
(a)
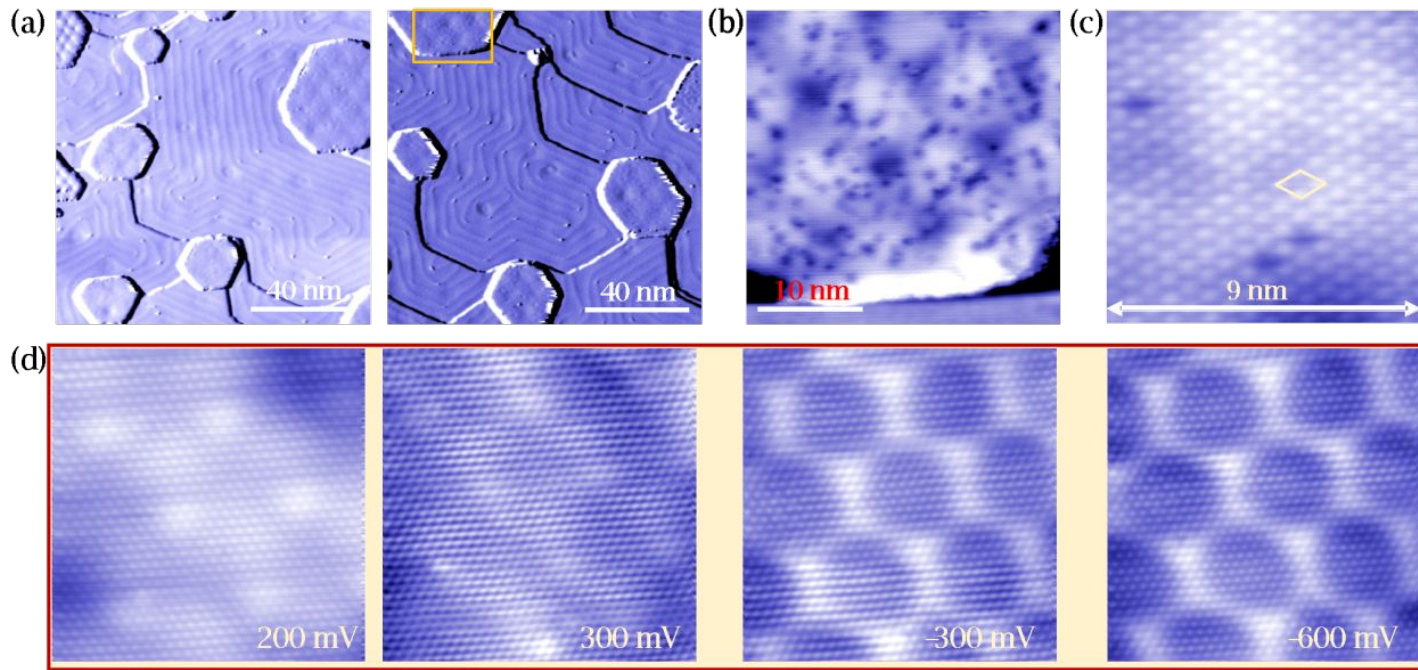

Fig. S8. Evolution of $\beta$-phase stanene and $\mathrm{Au}_{2} \mathrm{Sn}(111)$ Moiré pattern after annealing at 530 K. Scanning parameters: (a) 2 V, 100 pA (left); 1.8 V, 80 pA (right); (b) $500 \mathrm{mV}$, $300 \mathrm{pA}$; (c) $100 \mathrm{mV}, 800 \mathrm{pA}$; (d) from left to right: $200 \mathrm{mV}, 600 \mathrm{pA} ; 300 \mathrm{mV}, 600 \mathrm{pA}$; $-300 \mathrm{mV}, 600 \mathrm{pA} ;-600 \mathrm{mV}, 1.2 \mathrm{nA}$.

$530 \mathrm{~K}$ annealing induces severe desorption of Sn species but the evolution of two hexagonal phases. The first one exhibits a rhombus lattice of $0.54 \mathrm{~nm}$. The second case demonstrates a Moiré superlattice at negative sample bias with a lattice constant of 0.33 $\mathrm{nm}$. This value matches well with that of the $\mathrm{Au}_{2} \mathrm{Sn}(111)$.

(a)

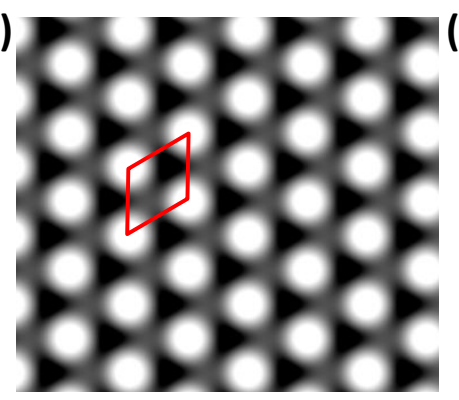

(b)

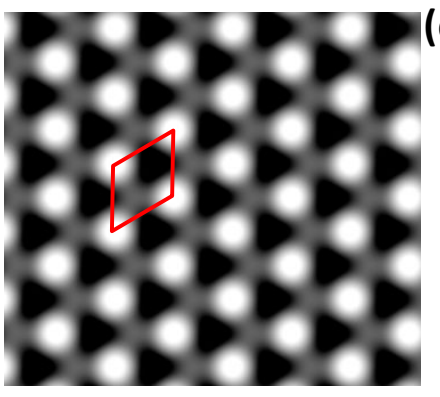

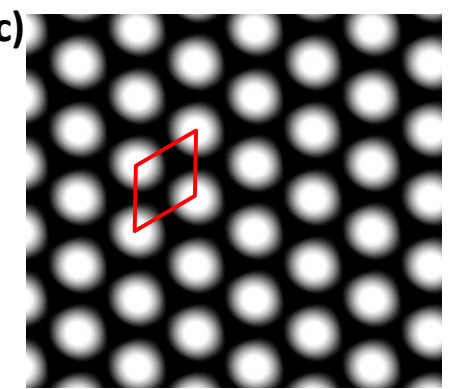

Fig. S9. Simulated STM topographic images of BLP-like stanene taken at different energy ranges: (a) $-1-1 \mathrm{eV}$; (b) $0-1 \mathrm{eV}$; (c) $-1-0 \mathrm{eV}$. 


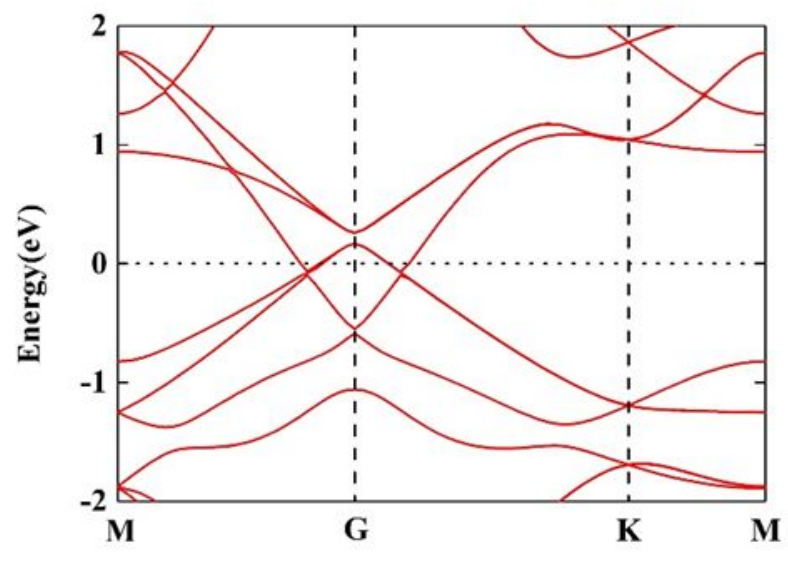

Fig. S10. Calculated band structure of freestanding stanene with a lattice constant of $0.5 \mathrm{~nm}$, corresponding to a $7.07 \%$ tensile strain.

(a)

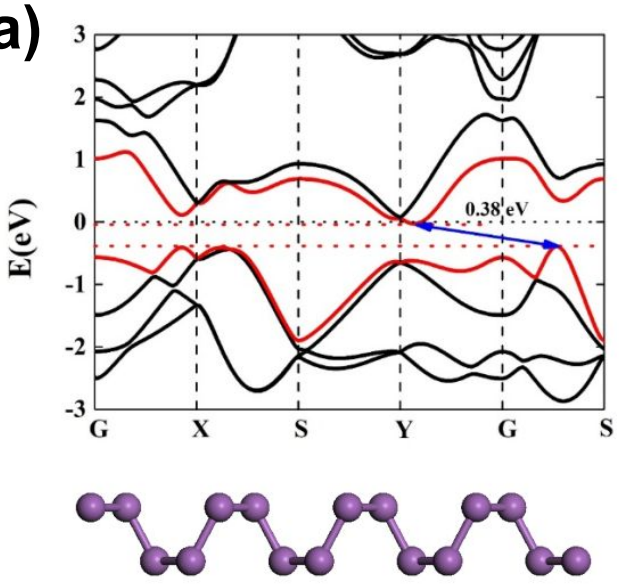

(c)

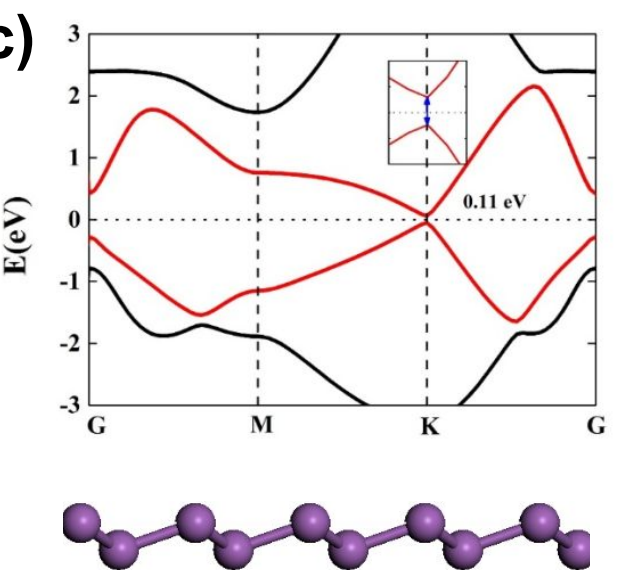

(b)
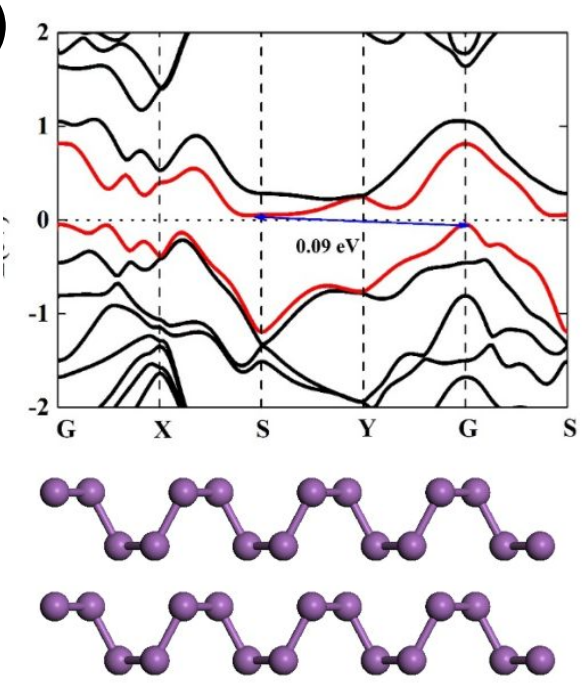

(d)

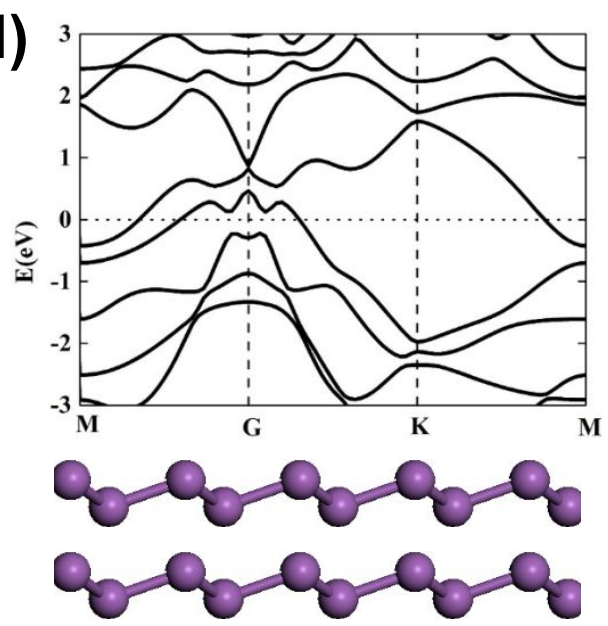

Fig. S11. Theoretically optimized atomic structures and band structures of $\alpha$ - and $\beta$ - 
stanene. (a) monolayer $\alpha$-stanene; (b) bilayer $\alpha$-stanene; (c) monolayer $\beta$-stanene; (d) bilayer $\beta$-stanene.

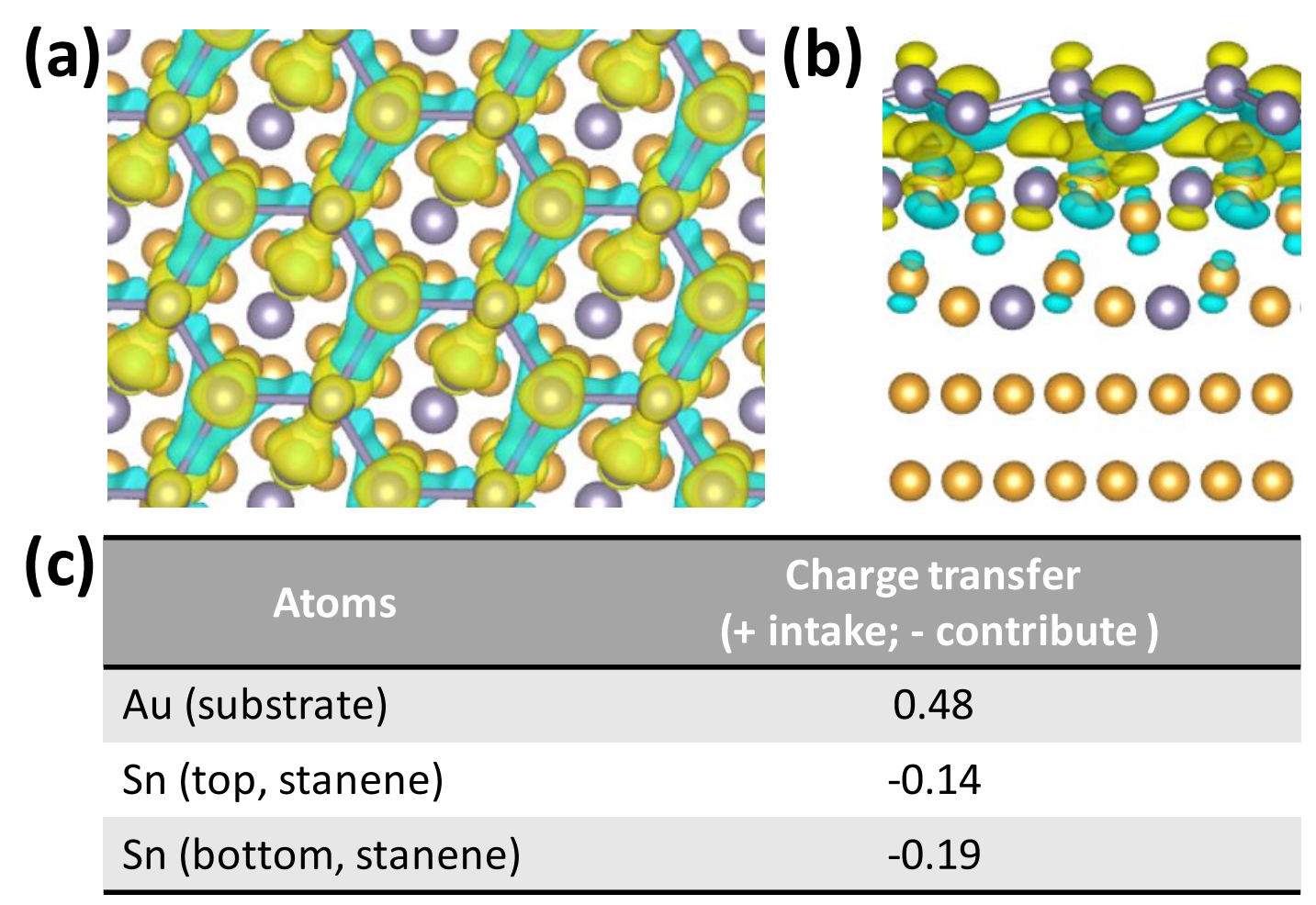

Fig. S12. (a) Top and (b) side views of the isosurface of the charge-density redistribution of BLP-like stanene on $\mathrm{Au}_{2} \mathrm{Sn}(111)$ surface. Blue and yellow colors indicate electron depletion and accumulation, respectively (isosurface value, 0.002 $\mathrm{e} / \AA^{3}$ ). (c) Calculated the number of electron per atom in the charge transfer system. 


\section{References}

(1) Si, N.; Shen, T.; Zhou, D. C.; Tang, Q.; Jiang, Y.; Ji, Q.; Huang, H.; Liu, W.; Li, S.; $\mathrm{Niu}, \mathrm{T}$. Imaging and Dynamics of Water Hexamer Confined in Nanopores. ACS Nano 2019, 13, 10622-10630.

(2) Giannozzi, P.; Baroni, S.; Bonini, N.; Calandra, M.; Car, R.; Cavazzoni, C.; Ceresoli, D.; Chiarotti, G. L.; Cococcioni, M.; Dabo, I. QUANTUM ESPRESSO: a Modular and Open-Source Software Project for Quantum Simulations of Materials. J. Phys.: Condens. Matter. 2009, 21, 395502.

(3) Perdew, J. P.; Burke, K.; Ernzerhof, M. Generalized Gradient Approximation Made Simple. Phys. Rev. Lett. 1996, 77, 3865-3868.

(4) Liu, C.-C.; Jiang, H.; Yao, Y. Low-Energy Effective Hamiltonian Involving SpinOrbit Coupling in Silicene and Two-Dimensional Germanium and Tin. Phys. Rev. B 2011, 84, 195430.

(5) Sadhukhan, P.; Pandey, D.; Singh, V. K.; Sarkar, S.; Rai, A.; Bhattacharya, K.; Chakrabarti, A.; Roy Barman, S. Electronic Structure and Morphology of Thin Surface Alloy Layers Formed by Deposition of Sn On Au(1 11 1). Appl. Surf. Sci. 2020, 506, 144606. 